\title{
Induced pluripotent stem cells-a new in vitro model to investigate $\alpha$-synuclein dysfunction in Parkinson disease
}

Researchers have used cells from a patient with Parkinson disease (PD) to generate induced pluripotent stem cells (iPSCs) that can be differentiated into neurons naturally overexpressing a-synuclein-a characteristic feature of PD. "One problem that has made progress in $\mathrm{PD}$ research more difficult is that the diseased tissue is not accessible during life," says Michael Devine, lead researcher of the study performed in collaboration with Tilo Kunath. The iPSC-derived neurons will enable in vitro investigation into the mechanistic basis of $a$-synuclein dysfunction and could eventually be used to test therapies for PD.

Triplication of the SNCA gene, which encodes $\alpha$-synuclein protein, causes a very rare but severe form of $\mathrm{PD}$, in which the levels of $\alpha$-synuclein in the brain are increased to double the normal levels. "We decided to create an iPSC model of SNCA triplication, because it is a fully penetrant cause of a very aggressive form of PD," explains Devine.

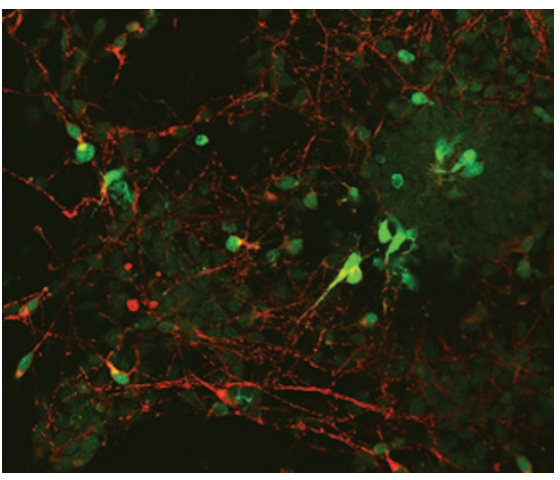

Confocal image of iPSC-derived neurons; $\alpha$-synuclein is stained in green. Courtesy of Dr Michael Devine.

Fibroblasts were isolated from a patient with the SNCA triplication and were then used to generate iPSCs. The stem cells were differentiated into midbrain dopaminergic neurons, and were found to contain double the quantity of $\alpha$-synuclein protein compared with neurons derived from a healthy relative of the patient. This finding confirmed that the model replicated the increased $a$-synuclein levels seen in the dopaminergic neurons of patients with the SNCA triplication.

Substantial variation was observed in the capacity of iPSC clones to generate neurons, highlighting the importance of deriving multiple clones from each patient to ensure that any differences observed are due to mutation and not clonal variation.

The research group is now using the cells to investigate how overexpression of a-synuclein disrupts the health of neurons. "Further ahead, these neurons could be used to screen for compounds that lower SNCA expression or inhibit the abnormal aggregation of $\alpha$-synuclein, which could form the basis for new treatments for PD," concludes Devine.

Katy Malpass

Original article Devine M. J. et al. Parkinson's diseas induced pluripotent stem cells with triplication of the a-synuclein locus. Nat. Commun. 2, 440 (2011) 